\title{
Microelectrode Analysis of Light Responses in the Brain of the Cricket (Gryllus domesticus)'
}

\author{
HUGH DINGLE ${ }^{2}$ AND STEPHEN S. FOX ${ }^{3}$ \\ Mental Health Research Institute, University of Michigan
}

\begin{abstract}
Electrical activity in response to light stimuli was recorded from the brain of the cricket (Gryllus domesticus) using stainless steel microelectrodes. Four basic types of elements were observed as follows: (1) units which registered ambient light intensity by frequency of firing as well as responding with transient changes in rate to stepwise increases or decreases in intensity; (2) units which fired at a higher frequency in dark than in light; (3) units which fired continuously at low level in light and responded with a transient high frequency burst to light off; and (4) units which responded with a brief burst to on and off, but tended to be "on-dominant" or "off-dominant." Also observed were synchronized spikes in mushroom body resfonding primarily to light off, but also on occasion to light on, and often accompanied by single unit responses. The units registering intensity are probably homologous with units showing similar properties recorded from the visual systems of several other arthropods and usually referred to as "sustaining units." On-off, off, and dark units are also known from other forms. The mushroom body light responses were similar to synchronized spikes recorded in cockroach mushroom body following antennal stimulation.
\end{abstract}

In recent years several studies have appeared dealing with visual responses in neurons of the arthropod central nervous system. Mostly these have been concerned with the behavior of units in the optic lobes or nerves (e.g., Burtt and Catton, '56, '60; Ishikawa, '62; Waterman et al., ' 63 , et. seq.), although there have in addition been some analyses of elements in ventral nerve cord (Burtt and Catton, '59; Suga and Katsuki, '62). More recently studies of the behavior of visual units in the protocerebrum of Lepidoptera (Blest and Collett, '65a, b) have contributed significantly to knowledge of visual events occurring in the arthropod brain between optic lobes and ventral nerve cord. The research reported in this paper also concerns visual responses in insect protocerebrum; the animal chosen was the house cricket, Gryllus (Acheta) domesticus.

The cricket was chosen for study primarily for three reasons: first, it is easy to obtain and to keep in the laboratory, making available a constant supply; secondly, preliminary information is available (Huber, '60, '63) on functions of the various regions of the cricket brain using direct electrical stimulation with which to compare electrophysiological findings; finally, the behavior of crickets has been studied (Alexander, '61) providing a basis of comparison with behavioral parameters.

In this study, stainless steel microelectrodes were used to record electrical activity. Light and other stimuli were employed, and single unit and evoked responses were observed in several brain regions to mechanical, electrical, and light stimuli. Spontaneous activity was also observed; this was not modifiable by any of the stimuli tested. The most frequently observed single unit responses, however, occurred in conjunction with light presentation. Light stimulation consisted simply of sustained light or darkness, turning the light on and off, or dimming and brightening. No attempt was made to use more complex stimuli as movement or flicker. The results discussed in this paper are confined to visual responses although, in addition, responses were seen to antennal and somatic stimulation (Dingle and Fox, '64, '66).

Received July 21, '65. Accepted Mar. 20, '66.

1 This research was done during the tenure of a U.S.P.H.S., N.I.M.H. postdoctoral fellowship (H.D.) and was supported by N.S.F. grant GB-1711 to $S$. S. Fox. For technical assistance we are grateful to Miss Bonnie Cross and Miss Joanna Wirble.

2 Present address: Department of Zoology, University of Iowa, Iowa City, Iowa.

3 Present address: Department of Psychology, University of Iowa, Iowa City, Iowa. 


\section{METHODS}

A. Preparation. Adults of both sexes of the house cricket, Gryllus (=Acheta) domesticus, were used in the experiments reported here. These were raised in the laboratory in plastic containers with a loose matrix of paper and wood shavings and were fed and watered ad libitum. The food was primarily "chicken laying mash" obtained locally from a commercial feed supplier.

At the beginning of an experiment a cricket was anesthetized with ether for one minute. The legs and wings were then removed and the animal pinned ventral side up in a wax bottomed dish. The sub-esophageal ganglion was exposed via an incision in the neck membrane and the labial, maxillary, and mandibular nerves cut in order to prevent, as much as possible, muscular movement within the head capsule. Usually the proximal portion of the labium was removed as well.

The animal was then pinned dorsal side up with its head inside a well of plasticene made complete by a strip of plasticene over the prothorax. The head was held in place with insect pins, one of which served as an indifferent electrode, penetrating the cuticle just medial and slightly posterior to the eyes and crossing at about the center of the capsule. The well was then filled with a perfusion fluid (Hoyle, '53) made isotonic by the addition of $0.1 \mathrm{M}$ sucrose until the head was covered; the insect could thus still respire as the spiracles were outside the plasticene well. All further surgery was performed with the head immersed in perfusion fluid. During surgery and the experimentation which followed, the perfusion fluid was changed at one to two minute intervals. The head was thus surrounded at all times by fluid at or near room temperature $(20-$ $23^{\circ} \mathrm{C}$ ).

A dorso-frontal incision was made beginning just medial to one eye and above the lateral ocellus proceeding across the epicranial plate to the opposite cye, then down into the antennary socket to the antennal scape, across below the median ocellus to the opposite antennary socket, and back to the point of origin. The flap of cuticle thus formed was lifted out with forceps and freed of all adhering muscle and other tissue. The underlying air sacs, tracheae, and fat were then removed to expose the brain lying just beneath. As much of the brain as possible was exposed including the antennal lobes and basal portions of the optic lobes. To hold the brain immobile, small pieces of tightly rolled tissue paper were inserted both anteriorly and posteriorly. And finally, just prior to the beginning of microelectrode penetration, the ocellar nerves were excised and sufficient of the perineural sheath removed to effect penetration in the areas desired.

B. Stimulation and recording. The source used for all light stimuli was a standard low voltage microscope lamp placed about $25 \mathrm{~cm}$ from the eye. Intensity was regulated with a potentiometer (and thus changes in wavelength also occurred) and calibrated with a MacBeth Illuminometer (Leeds and Northrup); the range was from near total darkness up to approximately 4,400 foot lamberts. Onoff stimuli were given by inserting by hand a cardboard square between the light source and the eye.

Recording microelectrodes were made by acid etching stainless steel insect pins (Clay-Adams size 00) according to the method of Green ('58) to a tip diameter of approximately $1 \mu$. They were insulated by dipping in lacquer (Insl-X E 33) to within 2 or $3 \mu$ of the tip; impedances were of the order of 1 to 3 megohms. Although of small amplitude (usually 40$80 \mu \mathrm{V}$ ) and with a somewhat noisy background, single unit responses were easily obtainable. The electrodes were mounted in either a Pfeiffer Type HP-2A or a La Précision Cinématographique Type $\mathrm{K}$ (with Type $T$ carrier) microelectrode drive. The latter instrument has a counter which allows a direct reading of penetration depth in micra. Further positioning of the electrode was determined by surface contours.

The recording electrode was connected via a cathode follower and conventional capacity coupled low level pre-amplifier to one input channel of a dual beam cathode ray oscilloscope. Records were photographed from a "slave" CRO screen with a moving film camera. The second beam of the CRO registered signals from 
a photodiode indicating onset and duration of the various light stimuli.

We attempted no histological location of electrode position, but approximate locations could be determined by surface contours and the depth of the tip which could be read directly from the counter on the La Précision Cinématographique microdrive. There was some dimpling of the brain surface upon penetration although this was very slight. Thus, precise localization of the electrode tip was not achieved, but we feel the approximations reported at least give a reasonable indication of the organization of the cricket brain with respect to visual responses observed.

\section{RESULTS}

A. Responses of visual units. Responses were recorded from the brains of 47 crickets during the present study from a total of 116 units. Of these, 64 showed responses to light. These light responses could be divided into the four basic types which are described below.
1. Sustaining units. In light these single unit spikes were characterized by a continuously and indefinitely maintained level of activity, the frequency of which increased as the light intensity was increased; at very low light levels these units failed to respond at all, while at the highest levels activity was characterized by a tendency to be patterned in bursts. As light intensity was increased by steps, there was a transient brief period of high frequency followed by a return to a level of activity higher than that observed previously. There was some indication that higher levels of sustained firing were obtained if intensity was increased in smaller increments. If intensity was decreased, there was a brief total inhibition of activity followed by a return to lower level. The behavior of these sustaining units is summarized by the single unit response shown in figure 1 .

In addition to the sustained light response, it was observed that the time course of a response to sudden onset of

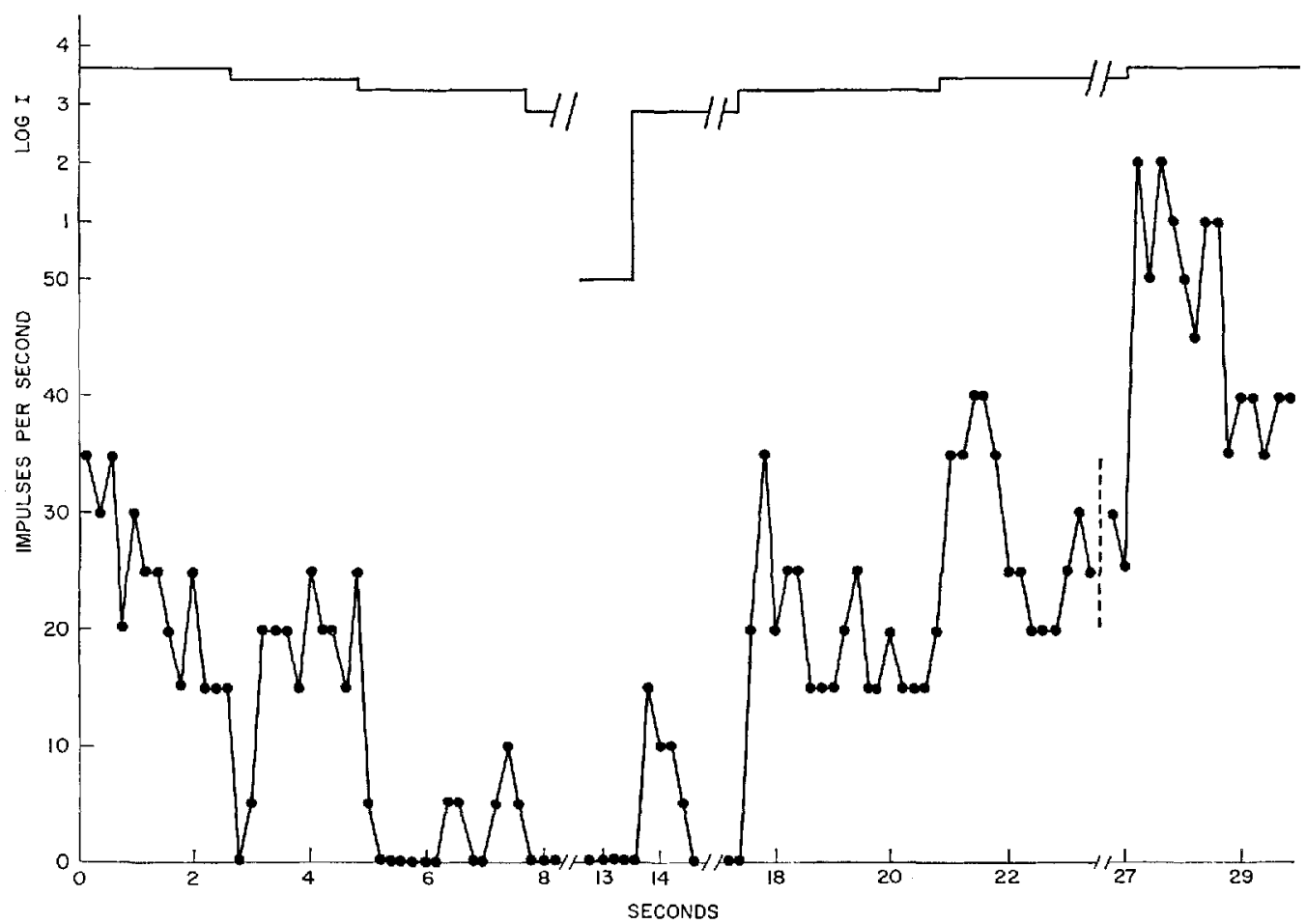

Fig. 1 Continuous plot of frequency against time for a sustaining unit. Upper line gives light intensity and changes in intensity while responses of the unit are given below. Sustaining units behaved in this fashion as long as preparation was healthy, often for several hours. 
illumination was a function of the duration of previous dark adaptation. The longer the time in the dark, the longer the period before the unit frequency would attain a base level of activity (fig. 2). Further, with sudden illumination the spike frequency oscillated for some time after the presentation of the stimulus. This oscillation of post-stimulus spike frequency is plotted in figure 2 , but is more clearly seen in figure 3 in which records are taken from two different preparations. In figure 3 the patterned oscillation is clear. These records show clear patterning for as long as eight seconds following the onset of the stimulus.
Sustaining unit responses were the most consistently observed discharges in all preparations, and once their approximate location was determined, they could be sampled regularly. They occur in a distinct region, probably a fiber tract, which extends from the optic lobe to the central body or its vicinity. Further, the visual impulses are transmitted primarily from the ipsilateral eye. Huber ('60) depicts three tracts entering the brain from the optic lobe. The sustaining units most probably occur in the middle of these three (number 2 of Huber, '63), based on the depth at which these units were found. The approximate locations within the

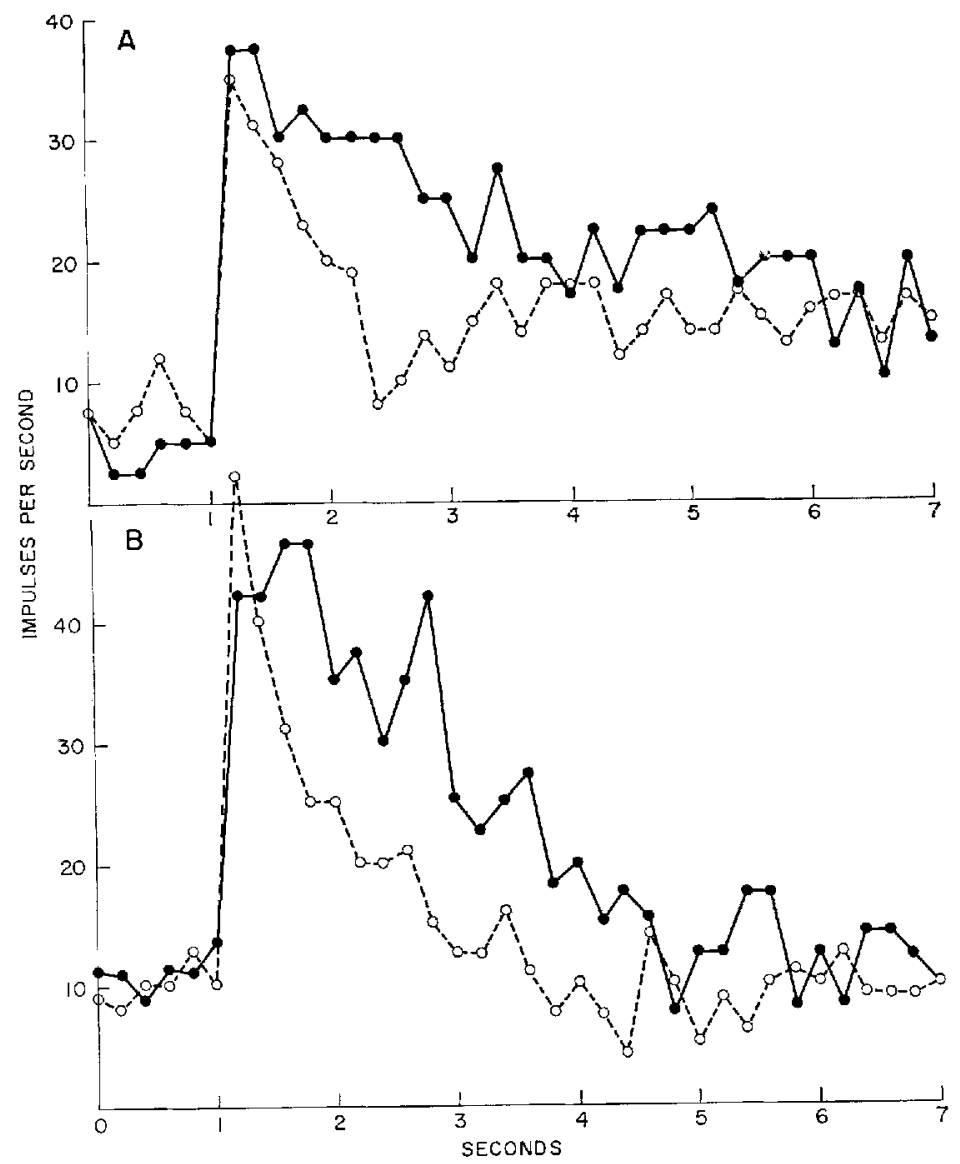

Fig. 2 Effect of duration of previous dark adaptation on responses of two sustaining units from two different preparations to light on. The time constant of the response period increases with increasing dark adaptation. In both graphs the response following the longer period of adaptation is indicated by the solid line and filled circles; the response following the shorter period by the broken line and open circles. 


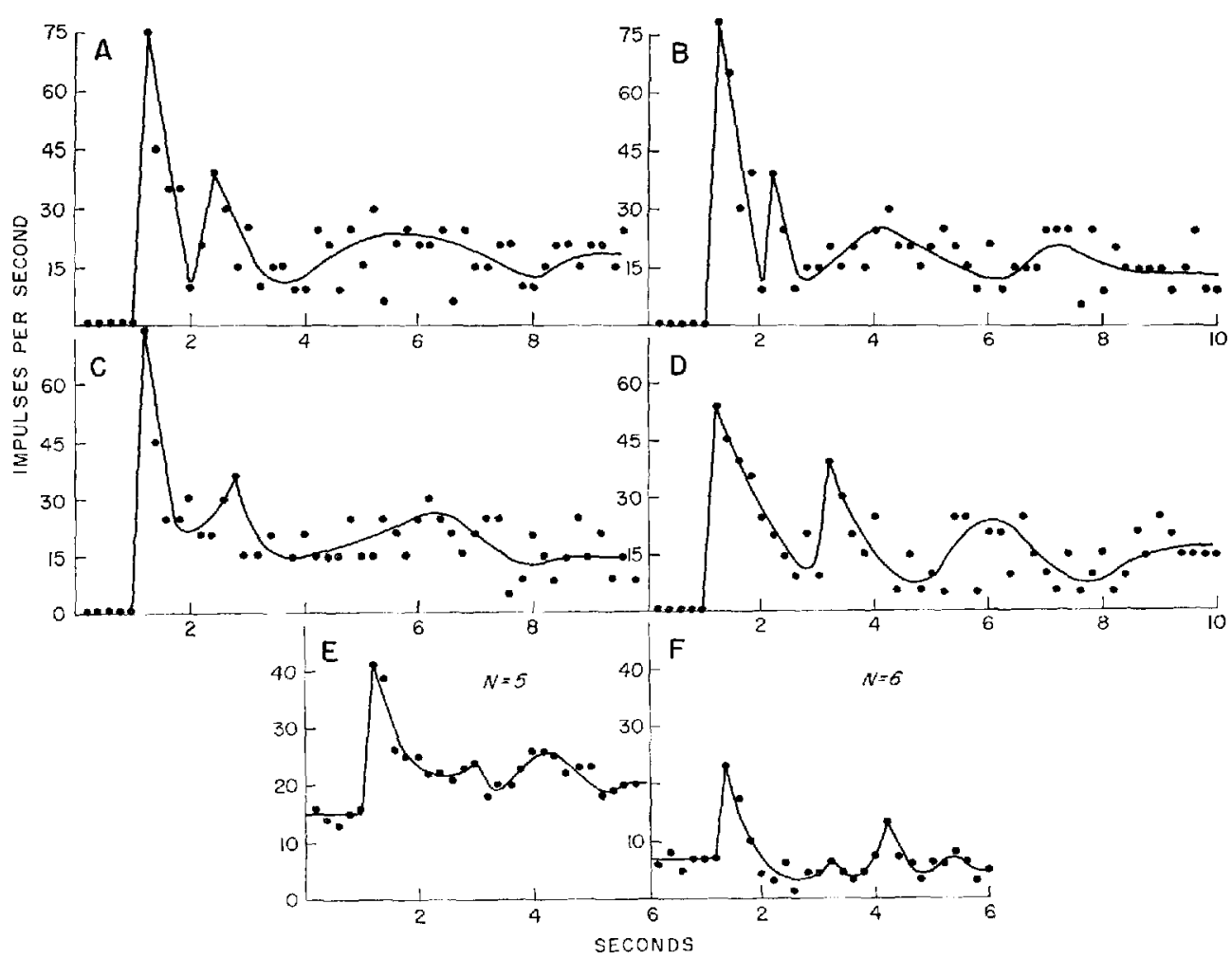

Fig. 3 Oscillations of sustaining units in response to increase of light intensity, A-D. Four responses of same unit kept in darkness before light turned on to full brightness. E, F. Two different units in a different preparation with responses to a brightened light averaged over five and six trials respectively.

brain of seven sustaining units from preparation C 19 are shown in figure 4; location data collected from other preparations is in general agreement with that indicated. There was no evidence that sustaining units occurred in any but the one region.

2. Dark units. Dark units showed a higher level of spontaneous activity in the dark than in light. Characteristically, if the light intensity was suddenly augmented, there was a marked transient increase in spike frequency followed rapidly by a return to a lower frequency than had been observed prior to the rise in intensity. With sudden darkening, there was again a transient burst. This was first followed by a cessation of activity for a brief period and then by a gradual climb to a level of activity higher than that in the light. The behavior of one of the three such dark units observed is summarized in figure $5 \mathrm{~A}$; these three observations of this type of unit were made on three different preparations. These units appeared to occur in fiber tracts coming from the optic lobe to the central region of the brain and were not observed in the same areas as the sustaining units, above, but their precise anatomical location is not known.

One other type of unit was observed similar to the above three. There were, however, some noticeable differences. The behavior of this unit is shown in figure $5 B$. With light off, there was the usual transient burst followed by a decline to a steady level of firing. With light on, on the other hand, there was total cessation of all activity for periods of at least eight seconds followed by a gradual rise to a level roughly equal to that present in the dark. These units were located deep within the brain (fig. 4) in an area quite far removed from that where sustaining and 


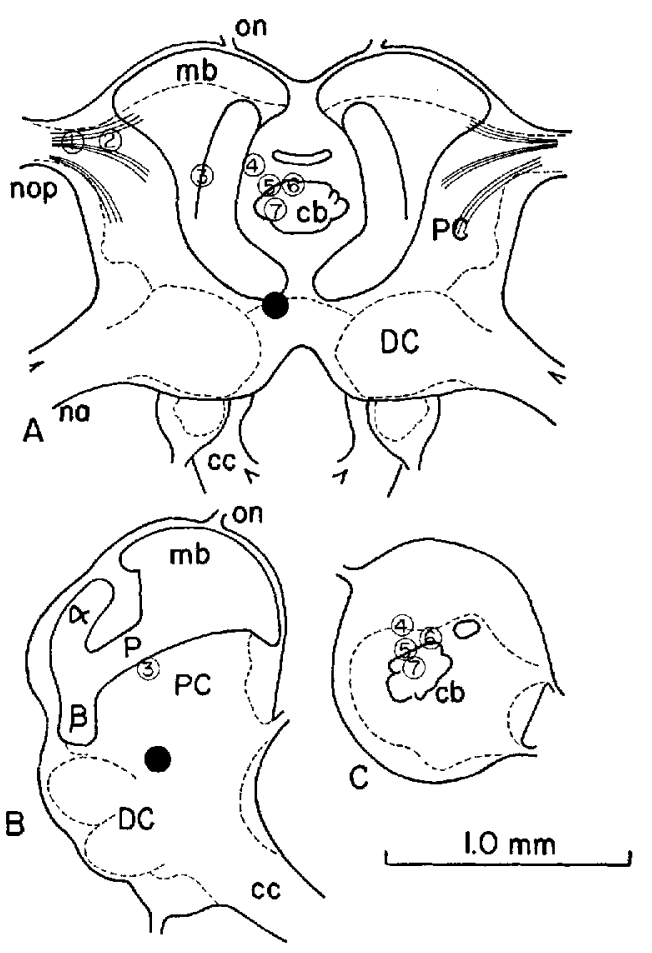

Fig. 4 Diagram of cricket brain (after Huber, '60). A. Frontal section. B, C. Sagittal sections taken, respectively, through mushroom body and through central body. Open numbered circles give approximate locations of sustaining units recorded in one preparation. Filled circle is approximate location of dark unit shown plotted in figure 5B and from a different animal. DC, deuterocerebrum; PC, protocerebrum; $c b$, central body; cc, circumesophageal connective; mb, mushroom body calyx below which lie the preduncle (P), alpha, and beta lobes; na, antennal nerve; nop, optic nerve; on, ocellar nerve.

probably the above dark units were located.

3. Light-off units. Only two light-off units in two different preparations were observed. One was located in the vicinity of the central body and anterior to it, while the other was located fairly deeply at the base of the optic lobe. These units fired continuously in the light and responded with a high frequency transient burst when the light went off. Following this burst, there was a rather rapid return to the level of firing observed in the light. No further change in activity was noted when the light again came on. 'The magnitude of the high frequency burst at off was a function of the duration of the previous period in the light. Figure 6 gives plots of the response of such a unit after three different periods of light adaptation and the record of the response after one of these periods.

4. On-off units. On-off unit responses were recorded from a variety of locations in almost all preparations including those which had deteriorated considerably. In the latter, they were invariably among the last units which could be observed. The on-off unit response consisted of a brief burst of spikes to either light on or light off with an even briefer burst, often of just a single spike, to the opposite stimulus. Consistently, a particular unit was either "off-dominant" or "on-dominant" with apparently more of the former. The typical on-off bursts consisted of 1-4 spikes although 17 were recorded in the burst of one unit (fig. 7A); the number of spikes in the response was dependent on the duration of the immediately preceding period of light or dark. Also, the latency of the response tended to shorten as the period of light or dark adaptation increased; this was not always obvious, however, and there were certain exceptions (fig. $7 \mathrm{H}$ ). In figure 7 are shown two on-off units, one on-dominant and one off-dominant, with different characteristics with respect to duration of response. The offdominant unit response is typical in duraation and number of spikes, but the ondominant unit response to light on is maximal for such a unit in both number of spikes and burst duration. These two units shown are from two different preparations.

Although these on-off units could be observed in quite deteriorated preparations, no evidence was found that they represent other types of units which have ceased to behave in their usual fashion. On-off units are also abundantly present in healthy preparations (based on the activity of the sustaining units) and are probably characteristic of normal conditions. In any case, these units are not simply aberrantly behaving sustaining fibers; under conditions of deterioration these latter showed a progressive decline in ability to respond until eventually they fired spontaneously and continuously and were completely unaffected by changes in light intensity which had previously been effective stimuli. 


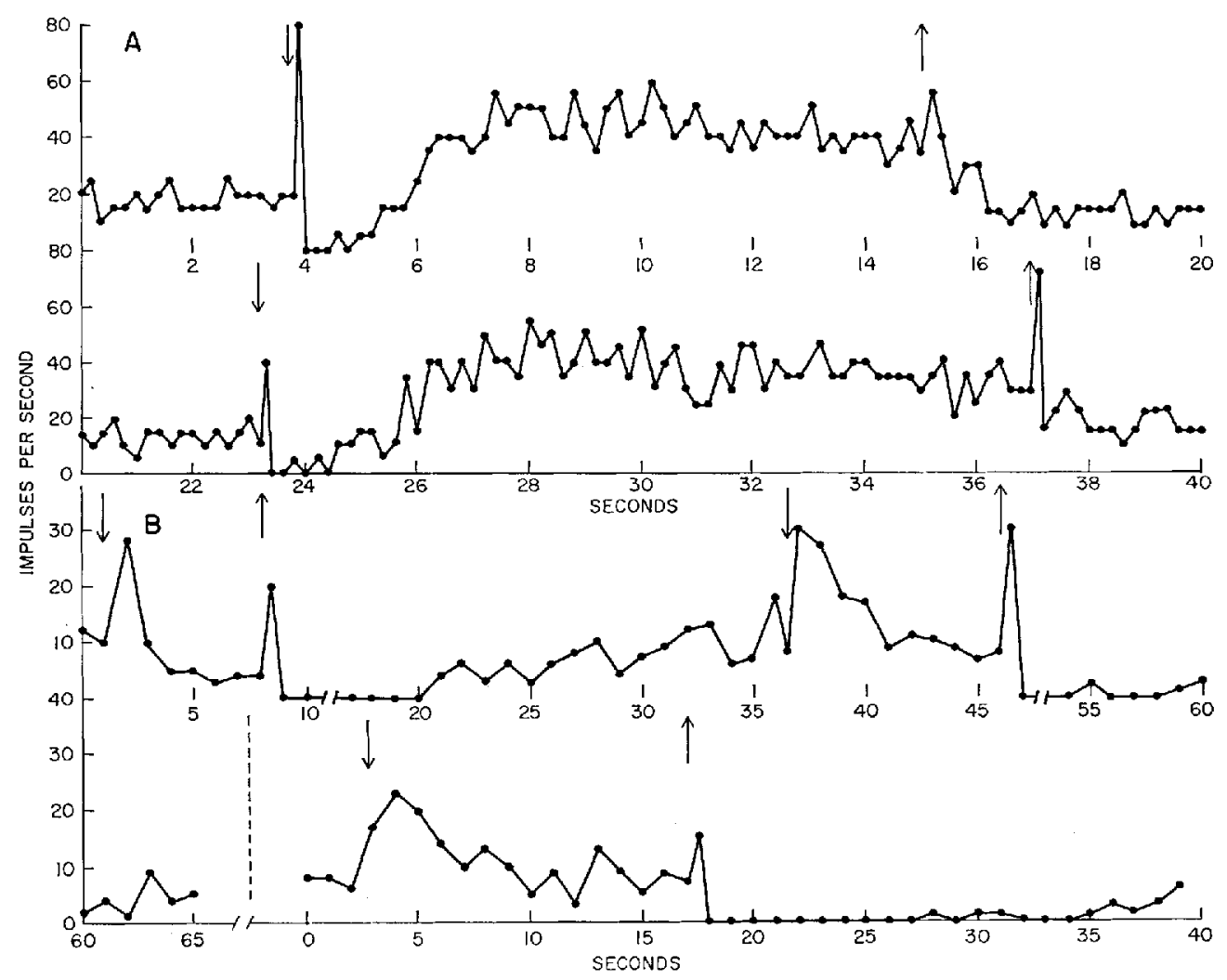

Fig. 5 Plots of dark units from two different preparations. A. Continuous plot of dark unit showing responses to dark, to light, and to light on (upward arrow) and off (downward arrow). $B$. Two continuous plots, separated by broken line, of a "dark unit" with somewhat different properties from that shown in $\mathrm{A}$. Note long periods of inhibition after light on followed by gradual return of activity, Location of this unit is given in figure 4 .

B. "Slow spikes." On addition to the classes of units responses described above, also observed were relatively slow (5-10 msec. duration) negative going long latency "spikes" which occurred primarily in response to light off, but on occasion also fired spontaneously. In some preparations there were similar responses to light on, but these were not always unambiguous. A distinct light on response is shown in figure $8 \mathrm{E}$ and $\mathrm{F}$. These spikes were of approximately three times the amplitude of the usual visual units (about $150 \mu \mathrm{V}$ ) although somewhat variable. There was some indication that amplitude of light off spikes increased after adaptation to light, but this was not consistent. The spikes sometimes occurred singly, but more often several times in a given response sequence. On other occasions fast single unit discharges were observed in conjunction with the slower potentials and were frequently carried on the slower wave. Figure 8 shows a response with but one or a few slow potentials and no units, while figure 9 shows a recording taken from a different placement in the same preparation with several slow potentials with accompanying faster units. As is seen in figure 9 and shown plotted in figure 10B, the number of spikes, both slow and fast, is a function of the preceding light adaptation time.

The parameter showing the most consistent effects of light adaptation was the latency of the spike if of single occurrence, or the latency of the first potential in a sequence. Mean latencies of responses from four different preparations are shown in figure $10 \mathrm{~A}$ plotted against adaptation in 


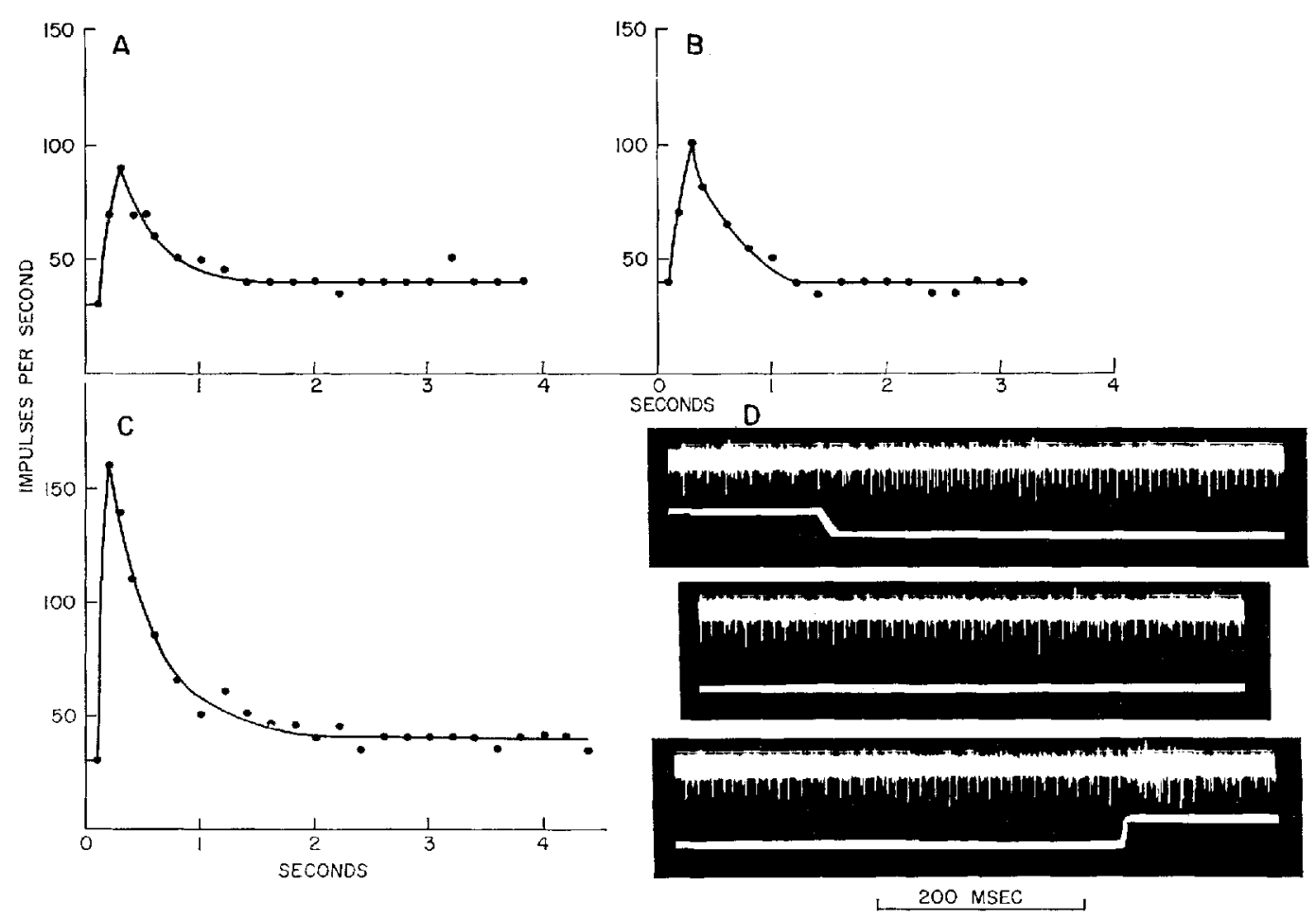

Fig. 6 Response of spontaneously firing unit to light off. A-C Plots of unit after four seconds, 5.5 seconds, and over 30 seconds, respectively, of previous light adaptation. Note increase in frequency response with longer light adaptation. D. Continuous photographs of response after 5.5 seconds light adaptation. Note also a second unit giving a small on burst. Lower trace in this and all succeeding photographs is output of photodiode aligned with eye. Up is light on; down light off.

light; an inverse relationship is clearly demonstrated. With lower intensity light, the latency had some tendency to increase although the data were somewhat inconsistent. A plot of latencies using low intensity light for one of the preparations is shown in figure $10 \mathrm{~A}$, and in this particular instance a shift was apparent. In general, however, a relationship between latency and intensity was not as clear as that for the reduction occurring as a result of increasing previous period of light.

No histological determinations were made of the anatomical location of the electrode tip in the recording of these slow spikes, but in general it appeared that these responses arose from the mushroom bodies (corpora pedunculata). These structures could be located from surface contours and the electrode placed on the surface of the brain above them. With only a slight penetration the slow poten- tials were seen, but they disappeared again with only limited further penetration of the electrode. When the electrode was again raised to its former level the responses reappeared. Penetrations slightly lateral or medial to points where responses were found failed to reveal them. From these observations it is most likely that the mushroom bodies are the site of origin, but the contributions of the various calyces or lobes cannot be stated precisely. In preparations where slow spikes were recorded at shallow depths toward the midline of the brain, it was possible upon deeper or more lateral penetrations to record as usual sustaining units registering differences in intensity. These were never observed in the vicinity of the slow spikes and vice versa.

In a number of preparations, there was an overall increase in activity with light on in those areas where the slow spikes 
A
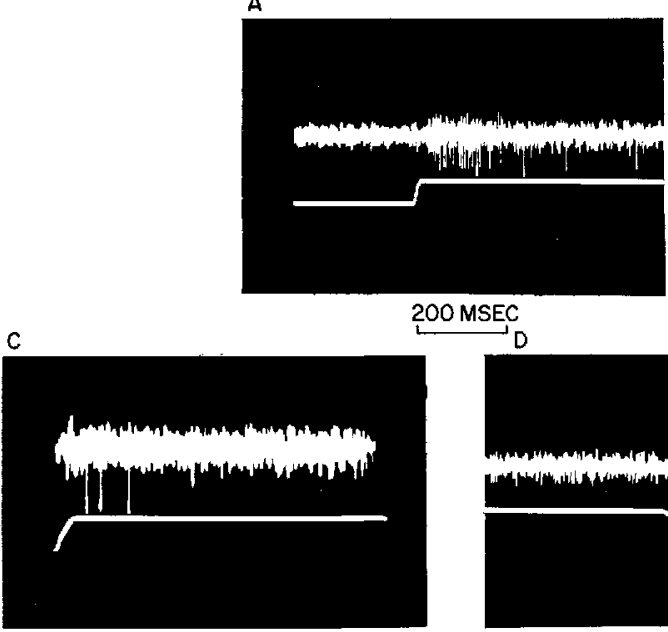

2OOMSEC

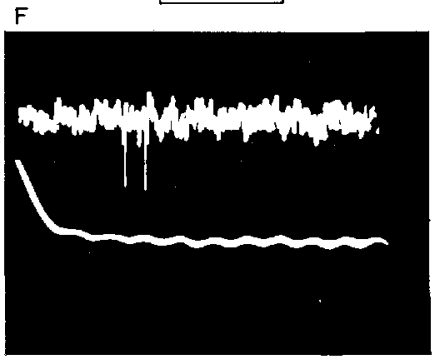

200 MSEC

OO MSEC
B

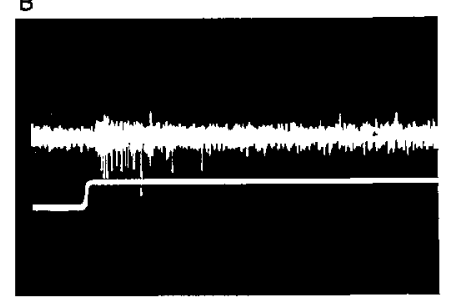

200MSEC

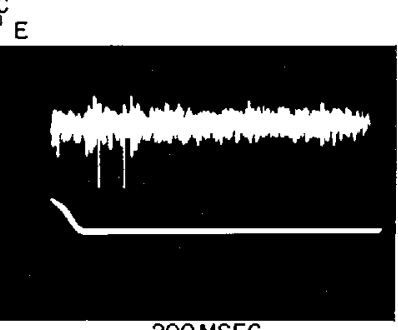

2OOMSEC

G

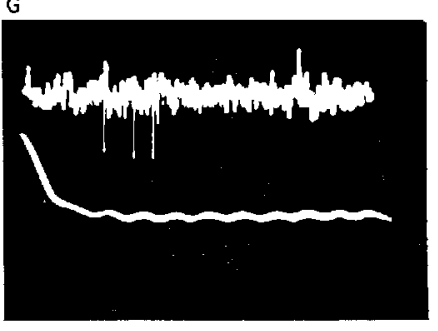

200 MSEC

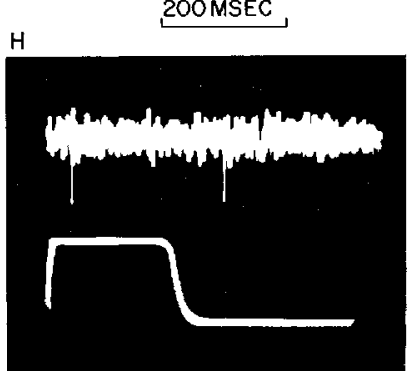

200 MSEC

Fig. 7 Responses of on-off units. A-C. On response of "on-dominant" unit following 30,6, and 2 seconds, respectively, of dark adaptation. D, E. Off response of same unit following 30 and 5 seconds of light adaptation. F, G. Off responses of "off-dominant" unit following 5 and 15 seconds light adaptation; note increase in number of spikes from 2 to 3 (consistently observed) and latency shift. H. Same unit showing single on spike after one minute dark adaptation and single off spike following the very brief interval in light. Single spike with relatively brief latency seemed to be characteristic of off-response following a flash.

were recorded. This activity was quite similar in appearance to that reported for locust mushroom body with light on (Burtt and Catton, '59) which provides additional support for the view that the slow spikes recorded in this study originated from the mushroom bodies.

\section{DISCUSSION}

Of the activity observed in the cricket brain in response to light stimuli, that of the sustaining units bcars the closest resemblance to a primary receptor response. The barrage of spikes in these units as a result of onset of illumination is quite similar to the pattern reported by Hartline and his colleagues in the lateral eye nerves of Limulus (Hartline, Wagner, and MacNichol, '52; Ratliff, Miller, and Hartline, '58; Ratliff and Hartline, '59; Ratliff, '61). With an increase in light intensity both Limulus and cricket units exhibit a transient burst followed by continued activity, the frequency of which is a function of the level of intensity. Hartline, Ratliff, and Miller ('61) have also shown that a given fiber when under inhibitory influences from several neighboring ommatidia, usually responds with a brief period of oscillation (fig. 3). Such oscillation might be expected since the whole eye was illuminated, and a given afferent fiber would be subject to a good deal of inhibitory influence. The reliability and duration of 
A

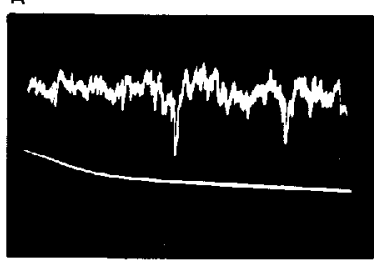

D

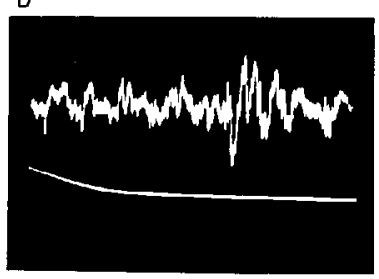

B

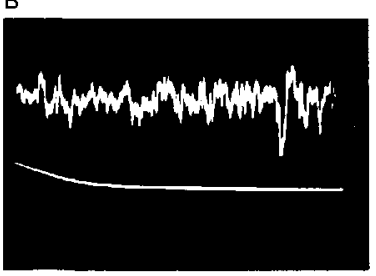

50 MSEC

$E$

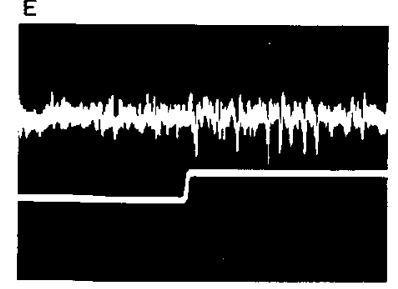

C

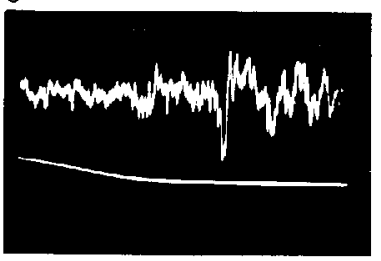

F

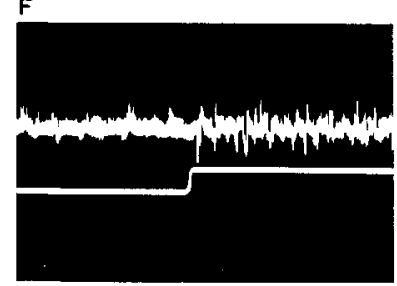

500 MSEC

Fig. 8 Synchronized activity recorded in mushroom body or immediate vicinity. A-D. Responses to light off after 5,10,20, and 40 seconds, respectively, of light adaptation. Note marked latency shift. In A, a "spontaneous" spike occurs before response to light off; this first spike is known not to be a light off response because responses after five seconds light adaptation occurred with latency of second spike in several additional trials. Filters on the pre-amplifiers were set to eliminate high frequency background after it was determined that no high frequency spikes (cf. fig. 9) were riding on slower wave (high frequency cut-off 250 cycles, low frequency 0.8 cycle). E, F. Responses to light on recorded in another preparation. Negative is down in all the above.

A

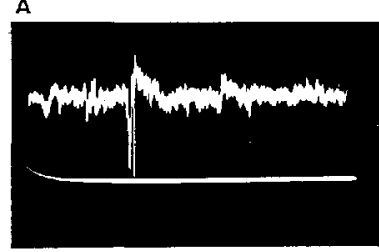

D

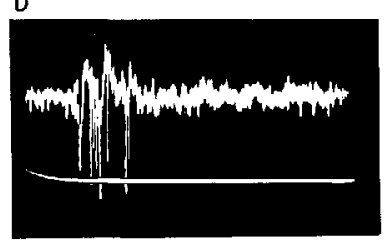

G

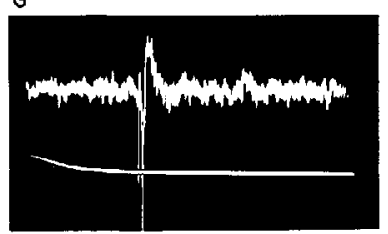

B

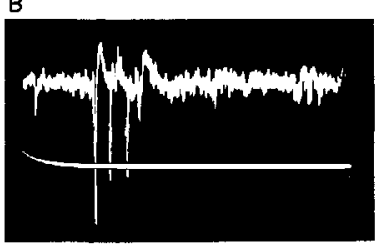

E

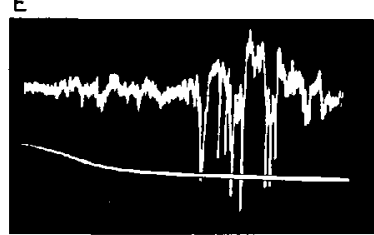

H

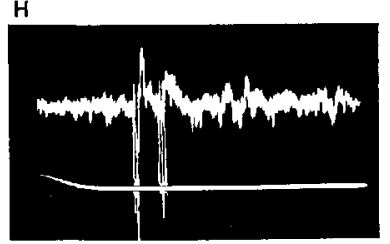

C

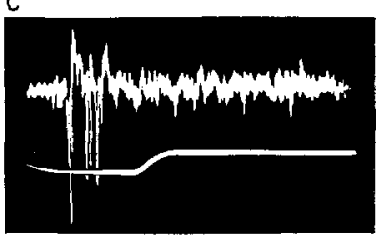

F

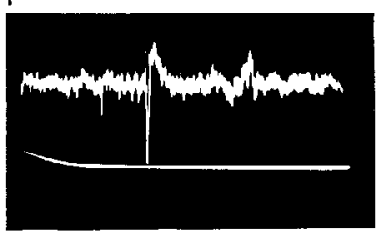

I.

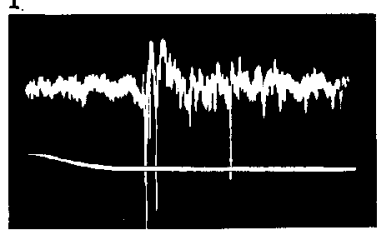

Fig. 9 Responses in mushroom body to light off in the same preparation as those illustrated in figure 8 , but with a different electrode placement. A-E. Responses after, respectively, 5, 10, 20,40, and 80 seconds of light adaptation showing latency shift and increase in number of spikes. Note absence of on response in C. E shows an expanded sweep; for this photograph only time mark equals 50 milliseconds instead of 100 milliseconds. F-I. Responses after, respectively, 5, 10, 20, and 40 seconds light adaptation in light that was about half the intensity of that used for A-E. Note generally longer latency and fewer spikes. Spikes in these traces retouched for photoduplication. 


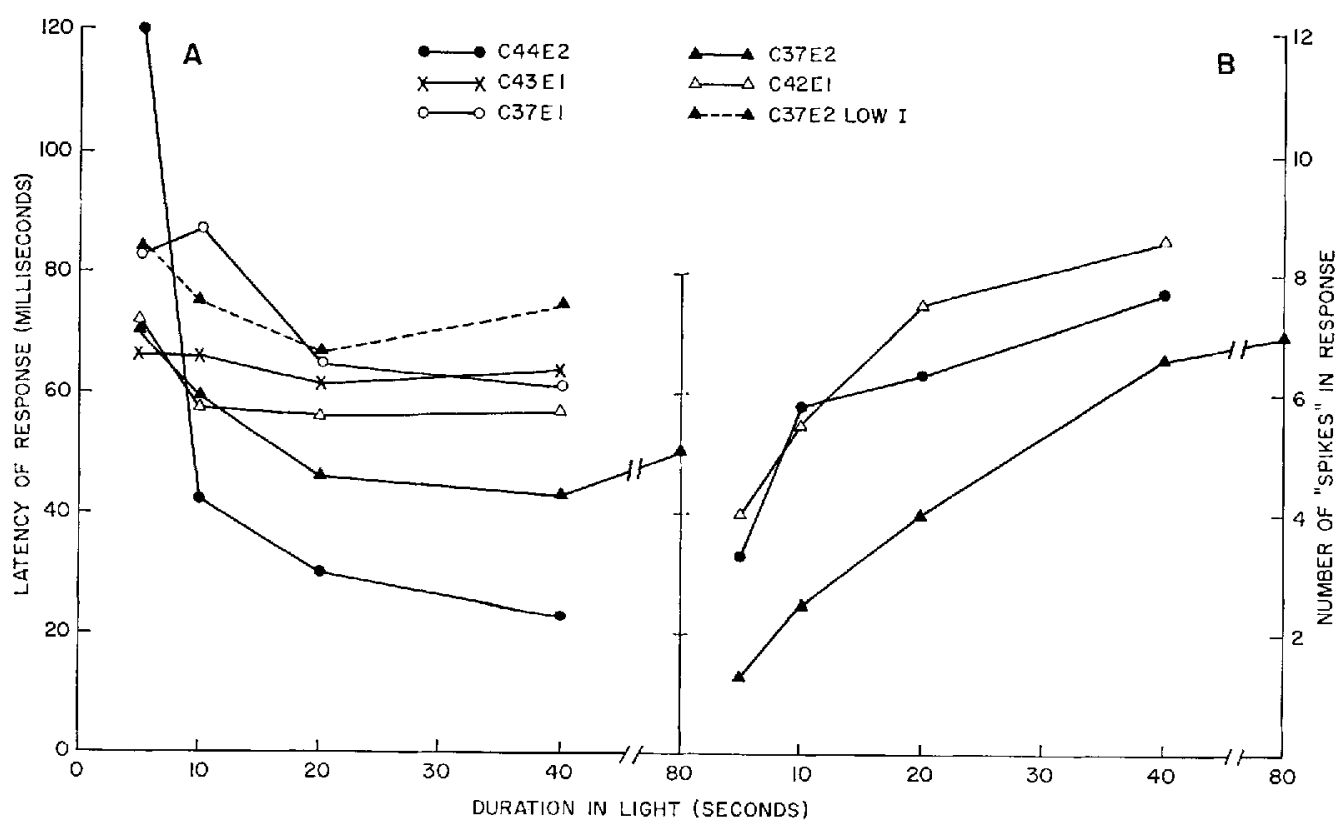

Fig. 10 plots of mushroom body responses to light off in four preparations. Mean latencies in $A$ and mean number of spikes in the response in $B$. C37E1 is illustrated in figure 8, A-D, and C37E2 in figure 9.

the oscillations indicates that in the cricket sustained coding of a transient stimulus occurs for at least eight seconds, about double the duration of oscillation noted for the response in Limulus ommatidia. Similarity to Limulus lateral eye nerve responses was also noted for sustaining units in the optic nerves of various Crustacea (Waterman and Wiersma, '63), in which units apparently represented integrated inputs from many ommatidia since they responded over a wide retinal area. Although not tested, the latter is probably also true of cricket units.

In addition to Limulus and cricket, sustaining units have been recorded from several other arthropod visual systems. They have been fairly extensively studied in various Crustacea (Waterman and Wiersma, '63; Waterman, Wiersma, and Bush, '64) where the tendency to fire in bursts to high intensity light, as well as the other properties seen in the present study, has also been noted. They were also found in the optic lobe of Locusta (Burtt and Catton, '60) and the protocerebrum of several Lepidoptera (Blest and Collett, ' $65 \mathrm{a}, \mathrm{b})$. In both cases they are referred to as "L-units." Blest and Collett have noted that they are readily sampled in tracts in the ventral anterior region of the brain passing between optic lobes and the medial lobe of the protocerebrum. The responses were unilateral. Quite similarly behaving units have been recorded in the optic lobe of the silkworm moth, Bombyx, (Ishikawa, '62), in the ventral nerve cord of the grasshopper, Gampsocleis (Suga and Katsuki, '62), and in the water beetle, Dytiscus (Bernhard, "42), recorded with surface electrodes between brain and optic lobe. With the exception of those in Limulus and Gampsocleis, all the above units were recorded either in the vicinity of the second synaptic region or in fibers proximal to all the synaptic regions; it, therefore, seems likely that they are from homologous nervous elements. In Limulus, recordings were from ommatidial fibers which presumably synapse at least once either before or in, the second synaptic region; in Gampsocleis, since the records were from the ventral nerve cord, there is probably a synapse between the units seen and those in optic lobe and brain (but see Burtt and Catton, '59). The 
sustaining fibers studied in the Crustacean eyestalk (Waterman and Wiersma, '63); Waterman, Wiersma, and Bush, '64) are undoubtedly the equivalent of those we observed in the cricket, the only differences being that the cricket lacks a long optic nerve.

In general, the above mentioned studies do not involve evaluation of the response of sustaining units to dimming of the light, as opposed to turning it off, although cessation of firing abruptly in darkness or very dim light has been noted (Waterman and Wiersma, '63; Burtt and Catton, '60). In the present study, stepwise dimming of light (fig. 1) resulted in a transient abrupt decrease in impulse frequency often to the point of cessation followed by a return to

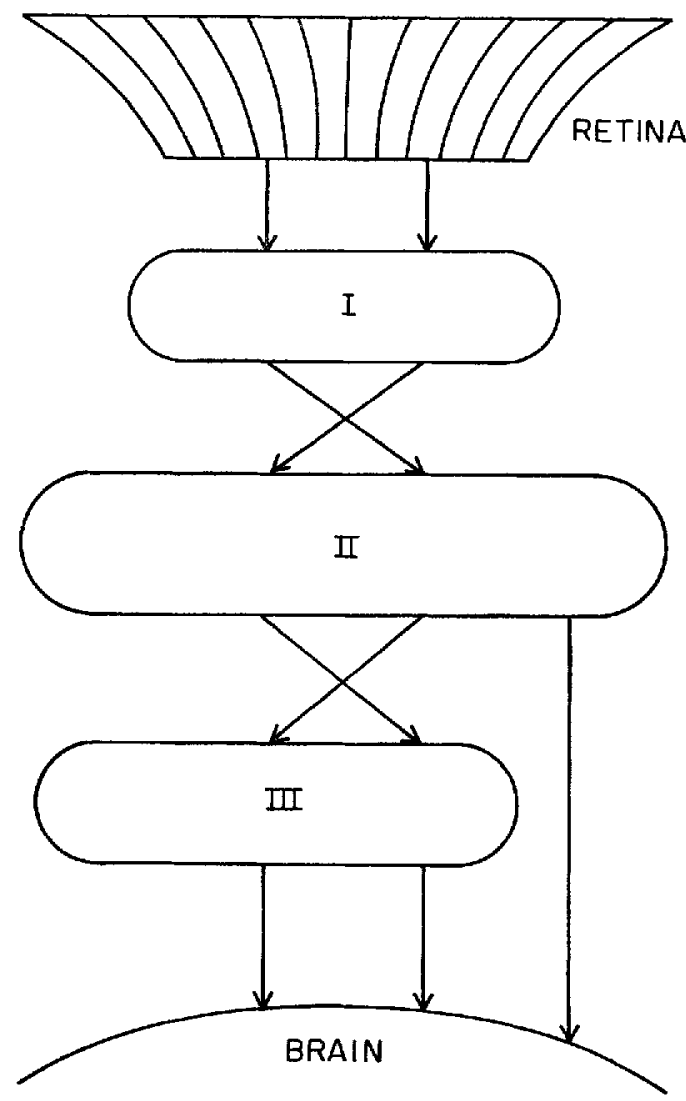

Fig. 11 Generalized diagram of retina and optic ganglia of insects: (I) first synaptic region (lamina ganglionaris), (II) second synaptic region (medulla externa), (III) third synaptic region (medulla interna). Arrows indicate pathways of nerve fibers. a level lower than that for the previous higher intensity light. The units are thus capable of signaling a change to either higher or lower light intensity as well as indicating the ambient level of illumination. The brief cessation of activity with dimming suggests that these units may be actively inhibited at that time. Blest and Collett ('65b) have demonstrated active inhibition of moth sustaining units ("L-units") by bursts of impulses coming from off fibers. These inhibitory impulses may be from the same or the opposite eye.

In view of the work of various investigators, outlined above, sustaining unit discharges seem to arise in the second synaptic region (medulla externa) of the optic lobe or in the cell bodies lying just outside this region (Burtt and Catton, '60). In insects, at least, sustaining units have not been seen until the electrode reaches that level (Burtt and Catton, '60; Ishikawa, '62) during penetration. Whether or not responses similar to those of the ommatidial fibers of Limulus will be found in insect ommatidial fibers remains to be seen; the only studies in insects of these peripheral units involved light flashes (Naka and Eguchi, '62) with the result that sustained activity would not have been noted even had it been present. Frequency, however, was shown to vary positively with intensity except at higher levels where inhibition occurred.

From the second synaptic region the sustaining fibers pass into the brain, but it is not known if they synapse in the third synaptic region (medulla interna). Certain large fibers bypass this structure and activity has been recorded from them by Burtt and Catton ('60); however, they did not indicate whether this activity included sustaining units. In the present work, the sustaining units were recorded in a distinct region extending from the optic lobes to the vicinity of the central body. Without histological verification, however, it cannot be said whether responses were recorded from the central body itself. The information transmitted by sustaining units also appears to reach the ventral nerve cord virtually unchanged (Suga and Katsuki, '62), although the steps in the process are not specified, and in addition is carried to the contralateral 
optic stalk (Wiersma, Bush, and Waterman, '64; cf. also Burtt and Catton, '56). It is clear that the information is carried to many integrative sites within the central nervous system (see Bush, Wiersma, and Waterman, '64).

The most prominent units other than sustaining units were those exhibiting brief discharges to "on" and "off;" this type of unit has been recorded in all levels of the arthropod central nervous system below the primary receptor units, as would be expected in view of lateral inhibition at the periphery (Ratliff, Miller and Hartline, '58). These were found in several locations within the brain, but separate regions specific for these responses could not be distinguished. Sustaining units which have been strongly stimulated are modified and show on-off activity (Waterman and Wiersma, '63), but no evidence has been found of such a shift in behavior in the cricket. Since these responses were regularly observed in healthy preparations, it seems that functional on-off units occur normally in a diversity of areas within the cricket brain, to such an extent in fact, that it is not clear whether they represent visual inputs or post-synaptic outputs from the various integrative areas. The tendency of on-off responses to be "on-dominant" or "off-dominant" has also been noted earlier.

The "off-units" that were recorded were more complex than the on-off units discussed above or off-units seen by other investigators. Off-units reported elsewhere tend to respond with short bursts while in the present case the units fired spontaneously in the light and showed a transient burst folllowed by a return to a base level slightly above that in light (fig. 6 ). The duration and latency of the transient increase in frequency was determined by the state of adaptation, a characteristic also of the above on-off units. There is some similarity of these "off-units" to the "D-units" found in the optic lobe of the locust (Burtt and Catton, '60), but the latter produced bursts to on as well as off. Locust D-units were also the most frequently observed whereas the cricket off-units were seen only rarely. It may be, therefore, that the two responses do not in fact come from homologous fibers.
The unit responses distinguished here as "dark-units" are similar to those observed in moth protocerebrum (Blest and Collett, '65a). The response of figure $5 \mathrm{~A}$ most resembles the "D-units" of those authors, while that shown in figure $5 \mathrm{~B}$ closely matches the description of their "fast dark units." The latter type have also been noted in butterfly optic lobe (Swihart, '64). The dark units also resemble D-units from the second synaptic region of locust optic lobe (Burtt and Catton, '60), but in locust neither total inhibition by light (fig. 5B) nor the depression in activity following the transient burst to off (fig. 5A) were observed. Fast dark units have also been seen in the ventral commissure, although not in the ventral nerve cord (Blest and Collett, '65a). It would appear, therefore, that these responses arise in the optic lobe, probably at the second synaptic region, and in some cases at least are transmitted as far as the ventral commissure. It is not possible, however, to ascertain whether such activity is transmitted to integrative areas within the protocerebrum.

The duration of the large negative "slow spikes" recorded in the area of the mushroom bodies suggest synchronized activity in several individual units. Activity in the multiplicity of fine fibers of the neuropil region of the insect mushroom body (Vowles, '55) would produce such synchronized responses. Maynard ('56) using small glass pipettes $(0.5 \mu)$ also noted large spikes in cockroach mushroom body, apparently arising from synchronized activity in the processes of the globular cells, in response to antennal shock.

In this study, most common were several mushroom body responses with the number a function of degree of light adaptation (fig. 10) and to a lesser extent of light intensity; frequently there was accompanying unit activity. The negative polarity, duration, and latency of the spikes were consistent with observations (Maynard, '56) in cockroach mushroom body where there was also some unit activity and occasionally more than one firing of the synchronized spike per response. Spontaneous firing of the spike was apparently not observed in the cockroach. The mushroom bodies, therefore, appear to respond 
in similar ways to different stimulus modalities arriving presumably along different input channels. How these inputs are distinguished at the level of the individual cell is a question which has in arthropod nervous systems been very little explored.

The general question of differentiation of two or more signals at the cellular level is one which might also be raised in relation to the sustaining units observed in these studies. These cells or fibers were seen to respond to both transient change in intensity (increase or decrease of light intensity) and to the continuous ambient level of illumination. Although in the case of these sustaining units, the differentiation is an intramodal one rather than cross-modal as with the mushroom bodies, the mechanism of differentiation of the signals is obscure except to the extent that sustained frequency of firing corresponds to intensity level and brief rate changes signal intensity changes (fig. 1). What is apparent, however, is that representation of more than one stimulus quality does exist in the arthropod brain at the single unit level (cf. also Bush, Wiersma, and Waterman, '64) and most likely as in vertebrate brain (Fox and O'Brien, '65) some mode of temporal patterning of spikes differentiates the two signals. The demonstration of the existence in arthropod brain of such multifunctional representation at the single unit level provides a somewhat more advantageously simple system for studying temporal or patterned differentiation or coding in sensory systems.

Synchronized spikes in response to light on were clear in the cricket brain in only two preparations; in others they were often present, but were ambiguous. In appearance these responses were not unlike those observed by Burtt and Catton ('59) in locust mushroom body after light on although no prolonged cyclical activity after the stimulus ceased was seen. The paucity of occurrence suggests that these on units in cricket brain many be confined to a limited area; electrode placements in a variety of locations were tried without success in recording them. It is probably fair to conclude from information concerning electrode placement both in this study and in Burtt and Catton's, that this activity occurs in the calyces. In bumble bees, (Vowles, '55) tracts of fibers leading from optic lobe to calyx have been described, as have responses in the mushroom bodies to both optic and mechanical stimulation (see Huber, '65).

No mention has been made here of similarities (or dissimilarities) to vertebrate systems. These are not to be dealt with here since they are quite fully discussed (Waterman and Wiersma, '63, et. seq.) in studies of crustacean visual systems using movements and other more complex stimuli. It is clear, however, that no arthropod visual systems have been studied as extensively as have vertebrate (Hubel and Wiesel, '62; Maturana, Lettvin, McCulloch, and Pitts, '60) or molluscan systems (Young, '64).

\section{LITERATURE CITED}

Alexander, R. D. 1961 Aggressiveness, territoriality, and sexual behavior in field crickets (Orthoptera: Gryllidae). Behavior, 17: 130223.

Bernhard, C. G. 1942 Isolation of retinal and optic ganglion response in the eye of Dytiscus. J. Neurophysiol., 5: 32-48.

Blest, A. D., and T. S. Collett 1965a Microelectrode studies of the medial protocerebrum of some Lepidoptera I. Responses to simple, binocular visual stimulation. J. Ins. Physiol., 11: 1079-1103.

$1965 \mathrm{~b}$ Micro-electrode studies of the medial protocerebrum of some Lepidoptera II. Responses to visual flicker. J. Ins. Physiol., 11: 1289-1306.

Burtt, E. T., and W. T. Catton 1956 Electrical responses to visual stimulation in the optic lobes of the locust and certain other insects. J. Physiol, 133: 68-88.

- 1959 Transmission of visual responses in the nervous system of the locust. J. Physiol,, 146: $492-515$.

1960 The properties of single-unit discharges in the optic lobe of the locust. J. Physiol., 154: 479-490.

Bush, B. M. H., C. A. G. Wiersma and T. H. Waterman 1964 Efferent mechanoreceptive responses in the optic nerve of the crab, Podophthalmus. J. Cell. and Comp. Physiol,, 64: 327345.

Dingle, H, and S. S. Fox 1964 Unpublished observations.

1966 A response to cercal stimulation recorded in cricket brain. Nature, 210: 1050.

Fox, S. S. and J. H. O'Brien 1965 Duplication of evoked potential waveform by curve of probability of firing of a single cell. Science, 147: 888-890.

Green, J. D. 1958 A simple microelectrode for recording from the central nervous system. Nature, 182: 962. 
Hartline, H. K, F, Ratliff and W. H. Miller 1961 Inhibitory interaction in the retina and its significance in vision. In: International Symposium on Nervous Inhibition (E. Flory, ed.), pp. 241-284, Pergamon Press, New York.

Hartline, H. K., H. G. Wagner and E. F. MacNichol 1952 The peripheral origin of nervous activity in the visual system. Cold Spring Harbor Symp. Quant. Biol., 17: 125-141.

Hoyle, G. 1953 Potassium ions and insect nerve muscle. J. Exp. Biol, 30: 121-135.

Hubel, D. H., and T. N. Wiesel 1962 Receptive fields, binocular interaction and functional architecture in the cat's visual cortex. J. Physiol., 160: 106-154.

Huber, F, 1960 Untersuchungen über die Funktion des Zentralnervensystems und insbesondere des Gehirnes bei der Fortbewegung und der Lauterzeugung der Grillen. Z. vergleich. Physiol., 44: 60-132.

1963 The role of the central nervous system in Orthoptera during the co-ordination and control of stridulation. In: Acoustic Behavior of Animals (R. G. Busnel, ed.), pp. 440-488, Elsevier, Amsterdam.

- 1965 Neural integration (central nervous system). In: The Physiology of Insecta (M. Rockstein, ed.), Vol. II, pp. 333-406, Academic Press, New York.

Ishikawa, S. 1962 Visual response patterns of single ganglion cells in the optic lobe of the silkworm moth, Bombyx mori L. J. Ins. Physiol., 8: 485-491.

Maturana, H. R., J. Y. Lettvin, W. S. McCulloch and W. H. Pitts 1960 Anatomy and physiology of vision in the frog (Rana pipens). J. Gen. Physiol., 43, Suppl, 2: 129-175.

Maynard, D. M. 1956 Electrical activity in the cockroach cerebrum. Nature, 177: 529-530.
Naka, K., and E. Eguchi 1962 Spike potentials from the insect photoreceptor. J. Gen. Physiol., 45: 663-680.

Ratliff, F. 1961 Inhibitory interaction and the detection and enhancement of contours. In: Sensory Communication (W. A. Rosenblith, ed.), pp. 183-203, MIT Press, Wiley, New York.

Ratliff, F, and H. K. Hartline 1959 The responses of Limulus optic nerve fibers to patterns of illumination on the receptor mosaic. J. Gen. Physiol., 42: 1241-1255.

Ratliff, F., W. H. Miller and H. K. Hartline 1958 Neural interaction in the eye and the integration of receptor activity. Ann. N. Y. Acad. Sci., 74: 210-222.

Suga, N., and Y. Katsuki 1962 Vision in insects in terms of the electrical activities of the descending nerve fibers. Nature, 194: 658-660.

Swihart, S. L. 1964 The nature of the electroretinogram of a tropical butterfly. J. Ins. Physiol., 10: 547-562.

Vowles, D. M. 1955 The structure and connections of the corpora pedunculata in bees and ants. Quart. J. Microscop. Sci., 96: 239255 .

Waterman, T. H., and C. A. G. Wiersma 1963 Electrical responses in decapod crustacean visual systems. J. Cell, and Comp. Physiol., 61: 1-16.

Waterman, T. H., C. A. G. Wiersma and B. M. H. Bush 1964 Afferent visual responses in the optic nerve of the crab, Podophthalmus, J. Cell. and Comp. Physiol., 63: 135-156.

Wiersma, C. A. G., B. M. H. Bush and T. H. Waterman 1964 Efferent visual responses of contralateral origin in the optic nerve of the crab, Podophthalmus. J. Cell. and Comp. Physiol., 64: 309-326.

Young, J. Z. 1964 A model of the brain, Clarendon Press, Oxford. 